\title{
Indicadores de aprendizaje en educación media superior para la formación en desarrollo sostenible a través de entornos virtuales
}

Francisco Javier Acosta Collazo omo parte del análisis de la experiencia de aprendizaje en el contexto del curso de "Ecología y desarrollo sustentable" en el nivel medio superior de la Universidad Autónoma de Aguascalientes (UAA), se presentan los resultados de seguimiento a las evidencias de aprendizaje generadas en los entornos virtuales mediante la aplicación de indicadores de aprendizaje adaptados al curso de enero-junio 2020. La primera parte del artículo reafirma la importancia de la educación para el desarrollo sostenible en la actualidad, seguido de la trascendencia de los indicadores en el enfoque por competencias en el nivel educativo, así como la aplicación de estrategias educativas relacionadas con la enseñanza del desarrollo sostenible. La variada cantidad de información generada en los entornos virtuales permite el diseño y análisis de indicadores de aprendizaje que pueden proporcionar información clave para la planificación, seguimiento y evaluación en los cursos impartidos en la educación superior y media superior.

Palabras clave: desarrollo sostenible, competencia educativa, metodología del aprendizaje activo, indicador de aprendizaje.

\section{Introducción}

La perspectiva de sustentabilidad se identifica como parte del Ideario Institucional en el Plan de Desarrollo Institucional 2016-2024 de la UAA: "Impulsar el desarrollo sustentable, justo y equilibrado de nuestra sociedad: formando integralmente, en las diversas dimensiones humanas, a personas con perspectiva global que contribuyan de manera efectiva, comprometida y ética a la solución de las necesidades y problemáticas sociales..." (UAA, 2016, p. 1). De igual forma, en el Modelo Educativo Institucional (MEI) se pretende una educación de calidad responsable que "promueve la conciencia de la necesidad de un aprovechamiento racional de los recursos naturales, la protec-

《 La importancia de incorporar la dimensión ambiental y formativa que representa la educación para el desarrollo sostenible. $\gg$ ción del medio ambiente y la tolerancia hacia los sistemas sociales, políticos y religiosos" (UAA, 2007, p. 7).

Lo anterior, destaca la importancia de incorporar la dimensión ambiental y formativa que representa la educación para el desarrollo sostenible ${ }^{1}$ en los procesos de aprendizaje y enseñanza en los estudiantes de la institución.

\section{Competencias formativas en sustentabilidad}

El Centro de Educación Media (CEM) de la UAA, pionero en incorporar la educación ambiental formal del nivel medio superior en el Estado, aborda la dimensión del desarrollo sostenible en la asignatura Ecología y desarrollo susten-

1 Actualmente se identifica el término de "sostenible" al ser parte de los Objetivos del Desarrollo de la onv; y para efectos de simplificación, en el artículo se puede considerar como sinónimo de sustentable. 
table, como parte del actual currículo del bachillerato por competencias. Así, las competencias genéricas definidas en el programa son:

- Genera ideas innovadoras y aplica la creatividad en la solución de problemas.

- Promueve la mejora de la realidad social y natural.

- Asume una actitud de compromiso ante la problemática ambiental, y se involucra en acciones que contribuyen al desarrollo sustentable.

Es de destacar que la asignatura se vincula actualmente con los objetivos para el desarrollo sostenible que promueve la Organización de las Naciones Unidas (ONU), mediante una metodología activa que incorporan actividades formativas que promueven la transferencia de lo aprendido hacia el contexto del estudiante.

A grandes rasgos, el programa se divide en cuatro unidades que promueven la importancia de la educación ambiental como dimensión formativa y de participación social. La segunda unidad revisa los fundamentos de la Ecología como ciencia integradora y vinculada a cursos previos. La tercera unidad identifica los principales problemas ambientales en el contexto local, promoviendo las alternativas de solución, así como el tema del cambio climático. Finalmente, la cuarta unidad, desarrollo sustentable, visualiza la dimensión ambiental vinculada a los planes de desarrollo en dependencias gubernamentales. Finalmente, se concluye el curso con el tema de ética ambiental que considera las relaciones éticas entre los seres humanos, otros seres vivos y el ambiente natural.

En el contexto de la asignatura, las unidades III. problemas ambientales y $I V$. desarrollo sustentable, fueron desarrolladas en el entorno virtual debido a la contingencia sanitaria ante el coronavirus (COVID-19) e interrupción de clases presenciales. Por ello, se rediseñaron las experiencias de aprendizaje para el entorno virtual en un esquema simplificado que incluyó la unidad, competencias, ${ }^{2}$ actividades (estrategias), productos (evidencias) y criterios de evaluación.

En la Tabla 1 se presentan algunos ejemplos del diseño instruccional del esquema aplicado en las unidades mencionadas de la asignatura de Ecología y desarrollo sustentable en el período enero-junio de 2020. Posterior a la evaluación de los productos o evidencias, surgió el objetivo de considerar a la evidencia como indicador de los aprendizajes en un grupo conformado por 42 estudiantes.

2 Se podrá consultar el ámbito de las competencias disciplinares básicas en el programa del curso.
Para Ruiz (2010), la evidencia es una aportación que realiza el alumno en función de un criterio de verdad que busca la manifestación de una cosa de manera que no se dude de ella. Además, aclara que no es sólo un "documento físico", pues dentro del mismo están las evidencias que responden, o no, a criterios y además contiene información referencial como el de un producto integrador.

De tal manera que podemos centrar el enfoque de evaluación basado en evidencias integradoras que implican instrumentos distintos de evaluación a las pruebas o cuestionarios; sin embargo, se requiere de una metodología educativa innovadora que incluya la necesidad de adquisición de los saberes con referencias para la aplicación y construcción del conocimiento.

En el caso de la asignatura, el método de proyectos fomenta el trabajo colaborativo, así como la modificación de actitudes ante la problemática ambiental mediante el compromiso y acciones que contribuyen al desarrollo sustentable.

\section{Metodología del aprendizaje activo}

Para el desarrollo de las competencias, se requiere de una metodología centrada en los aprendizajes que considere, además de las necesidades y características propias de los jóvenes bachilleres, la explicación y aplicación de conocimientos de temas que pueden ser complejos como: cambio climático, modelos de desarrollo y ética ambiental. Por lo que es indispensable el diseño de estrategias didácticas.

La experiencia se llevó a cabo mediante prácticas educativas considerando el contexto de los estudiantes, en este caso, el confinamiento ante la pandemia y las formas de comunicación virtual durante este período del curso. A partir de la retroalimentación final del curso, los alumnos destacaron las actividades y tareas (individuales o grupales) que significaron un mayor aprendizaje significativo.

- Cálculo de mi huella ecológica.

- Lecturas y videos vinculados a los temas del programa.

- Ecotecnologías ${ }^{3}$ viables de aplicar en el hogar.

- Reflexiones sobre la "Carta de la Tierra".

3 En esta actividad, las ecotecnologías o ecotecnias, consideradas como innovaciones tecnológicas diseñadas para preservar y restablecer el equilibrio ecológico, incluye acciones como huertos urbanos, elaboración de composta, terrazas verdes, entre otros. 
Indicadores de aprendizaje en educación media superior para la formación en desarrollo sostenible a través de entornos virtuales

Tabla 1. Ejemplo de actividades que formaron parte del diseño instruccional en el curso de Ecología y desarrollo sustentable

\begin{tabular}{|c|c|c|c|c|}
\hline Unidad / Tema & Competencias & Actividad & Producto / Evidencia & $\begin{array}{l}\text { Criterio de } \\
\text { evaluación }\end{array}$ \\
\hline $\begin{array}{l}\text { III Problemas } \\
\text { ambientales } \\
\text { 3.3 Huella ecológica }\end{array}$ & $\begin{array}{l}\text { Transfiere } \\
\text { comprensiones } \\
\text { teóricas a situaciones } \\
\text { de la vida cotidiana }\end{array}$ & $\begin{array}{l}\text { Revisión de lecturas, } \\
\text { cálculo de huella } \\
\text { ecológica. } \\
\text { Elaboración de estrategia. }\end{array}$ & $\begin{array}{l}\text { Documento: } \\
\text { "Estrategia de } \\
\text { disminución de mi } \\
\text { huella ecológica" } \\
\text { en tarea. }\end{array}$ & Rúbrica 3.3 \\
\hline $\begin{array}{l}\text { IV Desarrollo } \\
\text { sustentable (Ds) } \\
\text { 4.1 Dimensiones del } \\
\text { concepto }\end{array}$ & \multirow{2}{*}{$\begin{array}{l}\text { Identifica las } \\
\text { dimensiones del } \\
\text { desarrollo sustentable } \\
\text { en su contexto }\end{array}$} & $\begin{array}{l}\text { Entrevista (en forma } \\
\text { virtual) a un profesionista } \\
\text { (actor social) sobre } \\
\text { el tema. }\end{array}$ & $\begin{array}{l}\text { Documento } \\
\text { "Entrevista: } \\
\text { percepción del DS" } \\
\text { en tarea. }\end{array}$ & Rúbrica 4.1 \\
\hline $\begin{array}{l}\text { IV Desarrollo } \\
\text { sustentable } \\
\text { 4.4 Ecotecnologías }\end{array}$ & & $\begin{array}{l}\text { Elaborar propuesta: } \\
\text { "Mi hogar, un lugar } \\
\text { sustentable". }\end{array}$ & $\begin{array}{l}\text { Tarea: "Mi hogar } \\
\text { sustentable" }\end{array}$ & $\begin{array}{l}\text { Lista de } \\
\text { cotejo } 4.4\end{array}$ \\
\hline
\end{tabular}

Fuente: tabla elaborada por Francisco Javier Acosta Collazo, a partir de las experiencias de aprendizaje de la asignatura Ecología y desarrollo sustentable.

Cabe aclarar que no sólo son actividades realizadas, pues cuentan con diseño formal como estrategia didáctica, incluyendo la importancia del aprendizaje significativo con perspectiva constructivista. Así, entre más nexos establezcan los estudiantes entre el conocimiento previo, su contexto y la nueva información recibida, mayor será su significancia. Se esperan, además, cambios de actitud y comportamientos en favor del medio natural.

De acuerdo con Novo (2006), la educación del siglo XXI debe considerar dos retos de la problemática englobada en el desarrollo sostenible: a) reto ecológico enfocado a la formación de las personas para reconstruir ideas y comportamientos hacia una relación de equilibrio con la naturaleza; y b) reto social que intenta transformar las estructuras de gestión y redistribución equitativa de los recursos de la Tierra.

\section{Indicadores de aprendizaje}

En cuanto a la evaluación continua, es importante señalar que gran parte de los productos o evidencias presentaron desempeños notorios considerados como indicadores. En la propuesta, se considera que no sólo la evidencia valorada a partir de los criterios de evaluación se visualiza como indicador único. En términos de calidad, en la edu- cación y de los entornos virtuales existe una variedad de indicadores que son fundamentales para el monitoreo y medición de impacto en base a la planeación.

En términos del enfoque educativo por competencias, los indicadores representan aspectos directamente medibles u observables, y se utilizan para operacionalizar las variables susceptibles de evaluación (Ruiz, 210, p. 50). Los indicadores pueden estar relacionados con la calidad de la educación promoviendo el seguimiento a estrategias y programas globales entre diferentes lugares o contextos, como el objetivo de desarrollo sostenible (oDs 4). ${ }^{4}$ En educación a distancia, se tiene una amplía gama de indicadores, pues la enorme información que se tiene en los entornos virtuales puede ser recolectada, analizada y presentada como parte de lo que se denomina analítica del aprendizaje. Sabulsky (2019) reporta una analogía, señala que es un registro de huellas digitales de quienes participan en los entornos digitales. Así, por ejemplo, la información que provee la plataforma de Moodle permite a los docentes dar seguimiento a cada una de las actividades realizadas (en aula virtual) por los estudiantes.

4 Objetivo 4: Garantizar una educación inclusiva, equitativa y de calidad, y promover oportunidades de aprendizaje durante toda la vida para todos (https://www.un.org/sustainabledevelopment/ es/education/). 
Tabla 2. Indicadores identificados en el curso Ecología y desarrollo sustentable

\begin{tabular}{llc}
\hline \multicolumn{1}{c}{ Categoría } & \multicolumn{1}{c}{ Indicador } & Valoración \\
\hline \multirow{2}{*}{ Resultados en calificación } & \multicolumn{1}{c}{ Promedio en los resultados del segundo examen parcial } & 6.37 \\
\cline { 2 - 3 } & Promedio en los resultados del tercer examen parcial & 8.64 \\
\hline \multirow{2}{*}{ Procesos } & Puntuación promedio en la evidencia "Modelos de desarrollo" & 74.40 \\
\cline { 2 - 3 } & Puntuación promedio en la evidencia "Mi hogar sustentable" & 88.69 \\
\hline \multirow{2}{*}{ Registros } & $\begin{array}{l}\text { Actividad registrada por el número de usuarios en el foro "Di- } \\
\text { lemas ético-ambientales" }\end{array}$ & 1371 de 43 \\
\cline { 2 - 3 } & $\begin{array}{l}\text { Actividad registrada por el número de usuarios en las instruc- } \\
\text { ciones semanales (1-5 de junio de 2020) }\end{array}$ & 56 de 25 \\
\cline { 2 - 3 } & $\begin{array}{l}\text { Actividad registrada por el número de usuarios en documentos } \\
\text { de apoyo (Lecturas tema 4.5) }\end{array}$ & 69 de 30 \\
\hline \multirow{2}{*}{ Impacto en el contexto } & Puntuación promedio del proyecto "Mi huella ecológica" & 94.40 \\
\cline { 2 - 3 } & Puntuación promedio del proyecto "Educación ambiental & 90.91 \\
\hline
\end{tabular}

Fuente: tabla elaborada por Francisco Javier Acosta Collazo, a partir de los resultados de las evaluaciones de los estudiantes del curso Ecología y desarrollo sustentable, 2020.

Como se puede sustentar, el concepto de indicador tiene diferentes criterios de acuerdo con su aplicación; sin embargo, para efectos de la propuesta, se interpreta como una herramienta que permite la medición de competencias educativas de manera cuantitativa, siendo un elemento relevante para el monitoreo de las evidencias.

En el contexto de la asignatura vinculada a la educación ambiental, se definieron nueve indicadores basados en cuatro categorías, como puede observarse en la Tabla 2. Las mediciones visualizan los resultados en la aplicación de los instrumentos de evaluación del conocimiento; puntuaciones en las evidencias como parte del proceso e indicadores relacionados con los registros. Estos últimos son importantes para el seguimiento de los estudiantes como fueron el número de usuarios que accedieron a las lecturas, y finalmente la aplicación de estrategias con el enfoque de proyectos que tuvieron un impacto en el contexto de los estudiantes vinculados a la dimensión ambiental.

《 En términos de calidad en la educación y de los entornos virtuales, existe una variedad de indicadores que son fundamentales para el monitoreo y medición de impacto.

\section{Conclusiones}

Ante la creciente problemática socioambiental y económica actual, los temas relacionados con el ambiente natural y desarrollo sostenible adquieren importancia relevante en el ámbito escolar. De tal forma que, como educadores en el siglo XXI, se deben diseñar estrategias que nos aproximen a un futuro sustentable tal como lo señala el Plan de Desarrollo Institucional 2016-2024 de la UAA. El contexto de la asignatura Ecología y desarrollo sustentable, así como el enfoque de competencias educativas en el CEM de la UAA, ha permitido diseñar y aplicar el aprendizaje experiencial como los proyectos de educación ambiental, y promover un enfoque reflexivo en los jóvenes del nivel medio superior.

Por otra parte, es claro que los procesos de aprendizaje en el ámbito didáctico contemporáneo y con estudiantes del nivel medio superior, representa retos mayores que demandan una diversidad de estrategias de aprendizaje como los métodos activos del aprendizaje situado. Aunado a lo anterior, las condiciones actuales de educación en línea aumentan la complejidad en los procesos del ámbito educativo; sin embargo, una de las ventajas en los entornos virtuales es el enorme potencial que se tiene en los repositorios de experiencias de aprendizaje e 
información, que puede ser empleado mediante el diseño de indicadores adaptados a las necesidades de los programas educativos.

Los indicadores de aprendizaje identificados en esta experiencia evaluaron resultados de la evaluación continua, desempeño del estudiante en los cursos mediante vínculos a las rúbricas, accesos a la plataforma virtual y vinculación con otros sistemas de indicadores como el impacto de la educación ambiental en el entorno de los estudiantes. Así como en otros indicadores de aprendizaje vinculados a los objetivos del desarrollo sostenible que plantea la ONU. Además, la aplicación y análisis de indicadores puede ser utilizada para demostrar la calidad en los programas educativos y visualización de datos mediante gráficos o dashboard.

\section{Metodologia del aprendizaje activo}

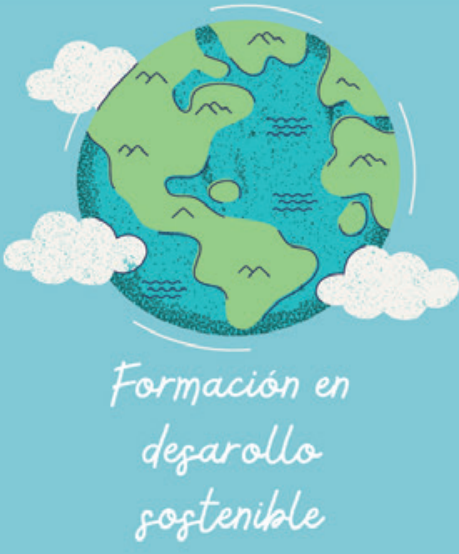

Se pretende una educación de calidad responsable que "promueve la conciencia de la necesidad de un aprovechamiento racional de los recursos naturales, la protección del medio ambiente y la tolerancia hacia los sistemas sociales, políticos y religiosos" (UAA, 2007, p. 7).

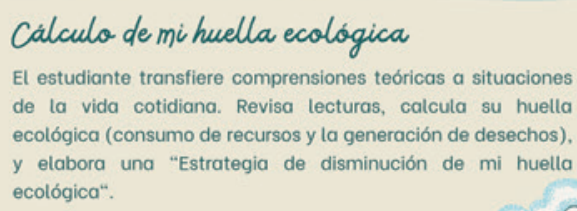

Ecotecnologiag viableg de aplicar en el hogar Innovaciones tecnológicas diseñadas para preservar y restablecer el equilibrio ecológico; incluye acciones como huertos urbanos, elaboración de composta, terrazas verdes, entre otros. El estudiante elabora la propuesta "Mi hogar, un lugar sustentable".

Reflexiones sobre la Carta de la Tierra.

Es un documento con dieciséis principios que impulsan un movimiento global. Se trata de un marco ético para las acciones encaminadas a construir una sociedad global más justa, sostenible y pacifica en el siglo XXI. El estudiante reflexiona los contenidos del documento.

\author{
El Centro de \\ Educación Media \\ de la UAA, pionero \\ en incorporar la \\ educación \\ ambiental formal \\ del nivel medio \\ superior en el \\ estado de \\ Aguascalientes, \\ aborda la \\ dimensión del \\ desarrollo \\ sostenible en la \\ asignatura \\ "Ecología y \\ desarrollo \\ sustentable", \\ como parte del \\ actual currículo \\ del bachillerato \\ por competencias.
}

\section{Fuentes de consulta}

Novo, M. (2006). El desarrollo sostenible: Su dimensión ambiental y educativa. Madrid: Pearsons.

Ruiz, M. (2010). Cómo evaluar el dominio de las competencias. México: Trillas.

Sabulsky, G. (2019). Analíticas de aprendizaje para mejorar el aprendizaje y la comunicación a través de entornos virtuales. Revista Iberoamericana de Educación, 80(1), 13-30. https://doi.org/10.35362/rie8013340

UAA. (2007). Modelo Educativo Institucional. En Correo Universitario, séptima época, (15). [Primera reimpresión], 29 de mayo de 2015. México: UAA. Recuperado de: https://bit.ly/2TPzsUo

UAA. (2016). Ideario de la Universidad Autónoma de Aguascalientes. Recuperado de: https://bit.ly/3mMUShz 